\title{
Statistical Simulation of Micro-Cavity Flows
}

\author{
Jian-Zheng Jiang, Jing Fan, and Ching Shen \\ Laboratory of High Temperature Gas Dynamics, Institute of Mechanics, CAS, Beijing 100080, China
}

\begin{abstract}
Two particle approaches, namely the information preservation (IP) method and the direct simulation Monte Carlo (DSMC) method, are used to simulate micro-cavity gas flows at low Mach numbers. In the continuum regime, the velocity profiles given by the IP and DSMC methods agree well with the Navier-Stokes solution of Ghia and the BGK solution of Su et al. The IP and DSMC results are in a satisfactory agreement at the Knudsen number $(K n)$ of 0.1 , and agree on the whole with each other but slightly differ from each other in certain details at $K n$ of 1.0. This small difference results from the strong singularity at the angle points that affects a larger and larger region as $K n$ increases. At very small Mach number the statistical scatter inherent in the DSMC method becomes serious, whereas the IP method still works well to obtain the flow fields and surface pressure distributions at a normal sample size.
\end{abstract}

\section{INTRODUCTION}

There is a great interest to understand in detail the aerodynamics of micro-electro-mechanical systems (MEMS). The characteristic length scale of MEMS is usually comparable to the mean free path of molecules, and the Knudsen number $(K n)$ is no longer small enough to be negligible, where $K n=\lambda / L, \lambda$ is the mean free path of the molecules, and $L$ is the characteristic length of the flow. Molecular-based numerical schemes, such as the direct simulation Monte Carlo (DSMC) method [1], are physically appropriate for this kind of gas flows where non-continuum, rarefied gas effects become important. In the DSMC method, macroscopic observable quantities, such as velocity and temperature, are obtained through averaging appropriate microscopic properties over a small space region. The simulated results are therefore inherently accompanied with statistical noise due to finite sample size. Some researchers [2-4] applied the DSMC method to micro-scale gas flows like those in micro-channels, and found it very difficult to obtain statistically convergent results under experimental conditions [5]. From equilibrium statistical mechanics, a signal-to-noise ratio in a dilute gas may be written as [6]

$$
\phi \equiv \frac{u}{\delta u}=M a \sqrt{\gamma N}
$$

where $u$ is the characteristic flow velocity, $\delta u$ is the statistical fluctuation, $M a$ is the Mach number, $\gamma$ is the specific heat ratio, and $N$ is the sample size. Micro-devices often operate at low $M a$, e.g. a typical flow velocity in micro-channel experiments [5] is about $0.2 \mathrm{~m} / \mathrm{s}$ that corresponds to $M a$ of $10^{-4}$. Relation (1) indicates that if we require the signal to be larger in order than the noise, e.g. $\phi=10, N$ has to be $10^{10}$. Such an enormous sample size is extremely time-consuming and beyond the capabilities of current computers [7].

There was a consideration [6] that when $M a$ is small enough for compressibility effects to be negligible, favorable relative statistical errors may be obtained by performing simulations at a $M a$ increased to a level where compressibility is still negligible. However, this is infeasible for rarefied gas flows MEMS is interested in, where the

CP663, Rarefied Gas Dynamics: 23rd International Symposium, edited by A. D. Ketsdever and E. P. Muntz (C) 2003 American Institute of Physics 0-7354-0124-1/03/\$20.00 
equality of both similarity parameters, $M a$ and $K n$, must be guaranteed simultaneously. Millikan's measurements of drag of a small sphere over the entire flow regime [8] may serve as a clear demonstration of this fact. The fitting formula of the measured data is dependent on two similarity parameters, $R e$ and $K n$. They can be transformed to $K n$ and $M a$ because of the relation [9],

$$
K n=1.255 \sqrt{\gamma}(\mathrm{Ma} / \mathrm{Re}),
$$

The number of similarity parameters is reduced to one only at extreme situations, i.e. $K n \rightarrow 0$ or $K n \rightarrow \infty$, corresponding to the continuum and free molecular limits, respectively.

In the past few years, the information preservation (IP) method [10-17] emerged as an alternative numerical technique to analyze subsonic, micro-scale gas flows. In this method [10], simulated molecules move through physical space and undergo collisions appropriate to the thermal velocities using the same algorithms and models as the DSMC method [1], while the macroscopic observable quantities, such as velocity and surface shear stress, are obtained through averaging appropriate physical information carried along with the simulated molecules. The physical information, updated by additional treatments [12-17], reflects the collective behavior of the enormous number of real molecules represented by each simulated molecule in the DSMC method, and therefore is not subject to the statistical noise caused by the thermal velocity. The IP method was successfully applied to benchmark problems, namely Couette flow, Poiseuille flow and Rayleigh flow [10,11]. The characteristic velocities in these flows ranged from $0.01 \mathrm{~m} / \mathrm{s}$ to $1 \mathrm{~m} / \mathrm{s}$, corresponding to $M a$ of $10^{-5}-10^{-3}$. The statistically convergent results obtained at a normal sample size of $10^{3} \sim 10^{4}$ were in excellent agreement with the exact solutions in the continuum and free molecular regimes, and those of linearized Boltzmann solutions $[18,19]$ and also with the experimental data [20] in the transition regime. Recently, Sun et al. [14] simulated low subsonic airflows past a micro flat plate using the IP method, and the calculated drag coefficient compared well with experimental data of Schaff \& Sherman [21], and Janour [22]. Sun \& Boyd [15] introduced an additional energy transfer model to update the information temperature that successfully solved the thermal Couette flow over the entire flow regime. Xie et al. [16] applied the IP method to varisized micro-channels at experimental conditions [23-25], and the simulated results were in excellent agreement with measured data of stream-wise pressure distributions and mass fluxes.

In this paper, we consider micro square-cavity flows that are quite simple in geometry but are singular in the regions near the angle points. Firstly, flow pattern around the angle points is analyzed, and the boundary conditions used in IP calculations are given. Then cavity flows of continuum to transition regimes are simulated using the IP and DSMC methods, and the results in the continuum regime are compared with the Navier-Stokes solution of Ghia et al. [26] and the BGK scheme solution of Su et al. [27]. In the slip and transition regimes the IP and DSMC results are compared between themselves. At extremely small Mach number the DSMC results give rise to serious statistic scatter whereas the IP results have smooth patterns at relatively not so large sample size.

\section{SINGULARITY OF THE ANGLE POINTS}

Cavity flows are driven by the top plate BC moving uniformly from left to right with a velocity $u_{w}$ (Fig. 1). The Knudsen number based on the side length of the cavity may be used to identify the degree of rarefaction of the gas. In the continuum regime with $K n<0.01$, the previous studies based on the Navier-Stokes equation [26] and 


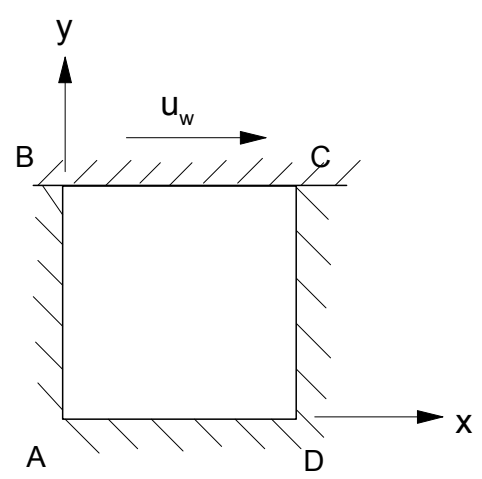

FIGURE 1. Schematic diagram of a square cavity gas flow.

the BGK scheme [27] showed that besides a primary vortex some secondary and additional vortices took place in the regions around the angle points. For rarefied gas flows concerned here, $R e$ is relatively small, and the flow complication mainly results from the velocity-slip phenomenon at surface that becomes significant as $K n>0.01$. For example, consider the angle point $\mathrm{B}$. The slip velocity means non-zero tangential components relative to the surfaces $\mathrm{AB}$ and $\mathrm{BC}$. On the other hand, these tangential components must vanish in approaching the surface $\mathrm{BC}$ and $\mathrm{AB}$ that are both impenetrable. Therefore, at the point $\mathrm{B}$, large pressure gradients in the $\mathrm{x}$ direction and in the reverse direction of y are essential. As shown later, this results in a discontinuity of pressure that considerably enhanced as $K n$ increases.

For two-dimensional gas flows, the implementation of the IP method was described in details in Ref. [13] that is employed in the present paper. The change of the information velocity due to a non-uniform pressure field may be written as

$$
\Delta u_{i}=-\frac{\Delta t}{\rho} \frac{\partial p}{\partial x_{i}}
$$

where $\Delta t$ is time step, and $\rho$ is the gas density. A third-order scheme is used to discretize the pressure gradient term. The pressure in the fictitious cell adjacent to the outer surface of cavity is determined through a linear interpolation. For rarefied gas flows with $K n>0.01$, the velocity slip at surface makes this treatment inaccurate in the angle-point regions with a radius of the mean free path. Further study is needed to improve the pressure boundary condition for the rarefied flows.

\section{MICRO-CAVITY GAS FLOWS}

An orthogonal coordinate system is employed in the IP and DSMC simulation (Fig.1): the origin is at A, and the $\mathrm{x}$ and $\mathrm{y}$ axes are along $\mathrm{AD}$ and $\mathrm{AB}$, respectively. The computational domain is divided into rectangular grids, and the sub-cell technique [1] is used to satisfy the requirement that the distance between a collision pair is to be smaller than the local mean free path. The Mach number is defined as $M a=u_{w} / a$, where $a$ is the sound speed of the initial stationary gas. The hard sphere model is employed to describe the interaction between molecules. All of the cavity surfaces are assumed to be diffusely reflecting, and to have the same temperature as the gas.

The first example considers a situation with $R e=100$, where $R e$ is defined based on $u_{w}$ and the cavity side length. Ghia et al. [26] and Su et al. [27] investigated this case using the incompressible Navier-Stokes equations and the BGK scheme, respectively. The BGK scheme solution was given at different Mach numbers [27]. When 
under $M a=0.15$, the relative difference in the peak velocity between the BGK solution and Ghia's data was less than $1 \%$. For $\mathrm{Re}=100$ and $\mathrm{Ma}=0.15$ relation (2) gives the corresponding $\mathrm{Kn}=2.48 \times 10^{-3}$. This indicates that the flow is in the continuum regime and the Navier-Stokes solution is reliable.

IP and DSMC calculations employ five million of simulated molecules, the time step is a half of the mean collision time, the number of cells is $100 \times 100$, and each cell contains $9 \times 9$ sub-cells. The DSMC calculation takes 60,000 sampling time steps to reduce the statistical scatter, 300 times larger than that used in the IP calculation. Figure 2a) shows the IP and DSMC profiles of the x-component of velocity along the vertical center line, which are in excellent agreement with the Navier-Stokes and BGK scheme solutions [26,27]. Figure 2b) compare distributions of the y-component of velocity along the horizontal center line given by the four above mentioned methods. In the whole they agree with each other, but differ are slightly at two places, i.e. at $x / L=0.25$ and 0.8 . The agreement between the incompressible Navier-Stokes solution and the three other results indicates that the similarity parameter is only $R e$ for this continuum flow limit at low subsonic regime. This is well known in conventional aerodynamics.

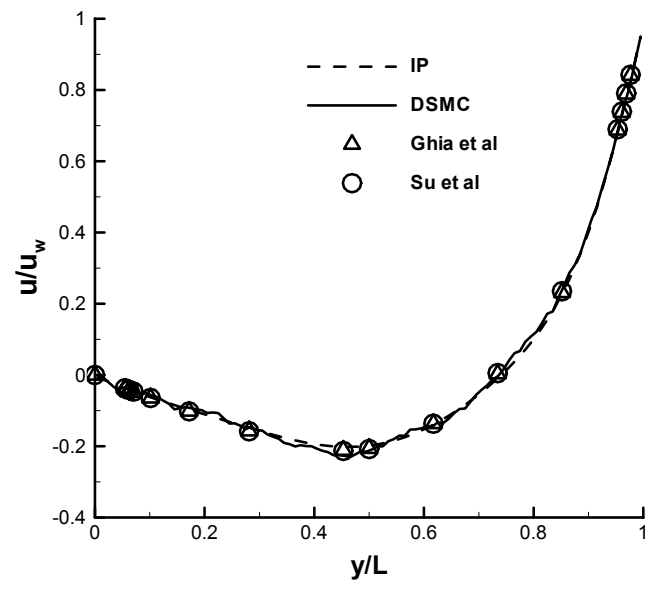

(a)

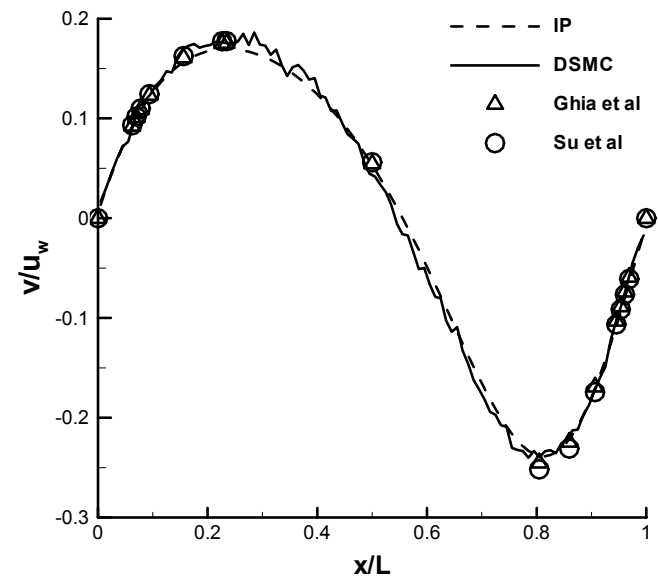

(b)

FIGURE 2. Comparison of the IP and DSMC velocity profiles with the Navier-Stokes solution [26] and the BGK scheme solution [27] at $R e=100$, and $M a=0.15$. (a) x- component of velocity along the vertical center line ; (b) y-component of velocity along the horizontal center line.

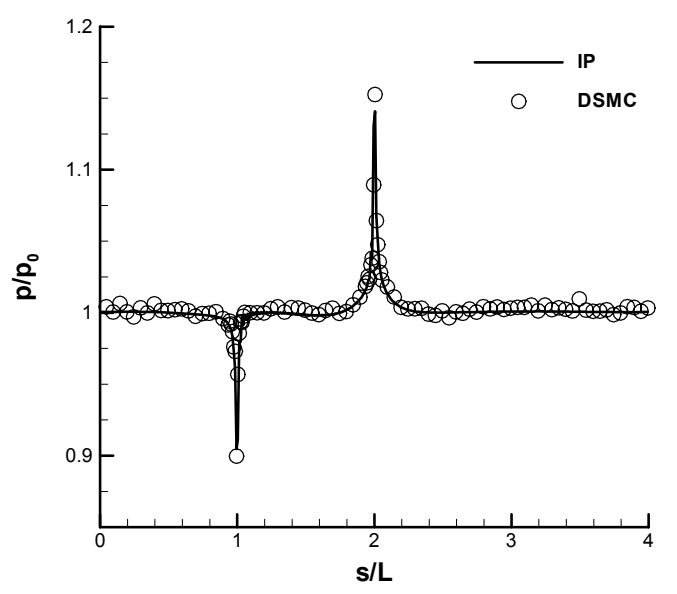

FIGURE 3. Normalized surface pressure distributions at $\mathrm{Re}=100$, and $\mathrm{Ma}=0.15$ given by the IP and DSMC methods. 
Figure 3 shows the IP and DSMC normalized surface pressure distributions along the cavity surface at $\mathrm{Re}=100$ and $\mathrm{Ma}=0.15$, where $\mathrm{s}$ is the distance from the point $\mathrm{A}$ in the clockwise direction. Except at the angle point $\mathrm{C}$ that corresponds to $s / L=2$, they agree well with each other.

Comparison of the IP and DSMC results for the case of $K n=0.1$, and $M a=0.14$ is shown in Fig.4. As can be seen, there is a satisfactory agreement between the velocity and normalized density $\left(\rho / \rho_{0}\right)$ fields, whereas the IP and DSMC normalized surface pressure distributions are slightly different between the angle points B and C. This deviation of the results from DSMC may attribute to the pressure boundary condition used in IP calculations that becomes inaccurate as $\mathrm{Kn}$ increases, especially at the angle points.

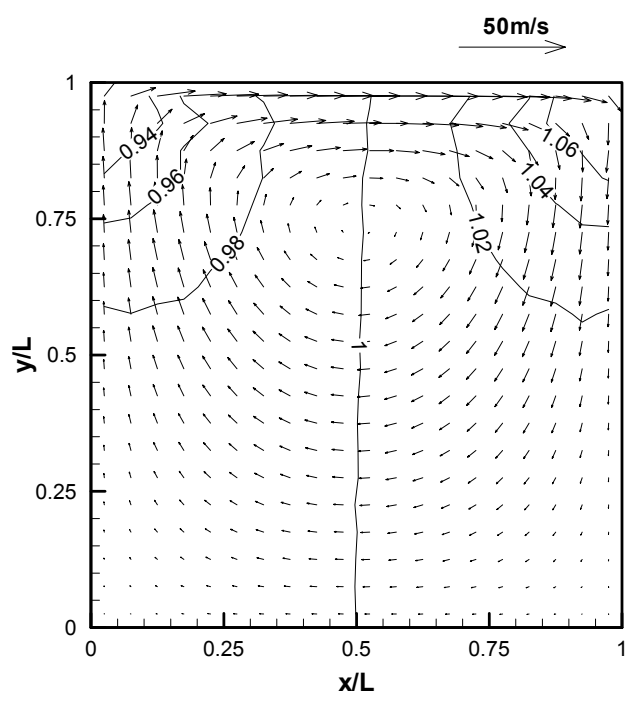

(a) IP

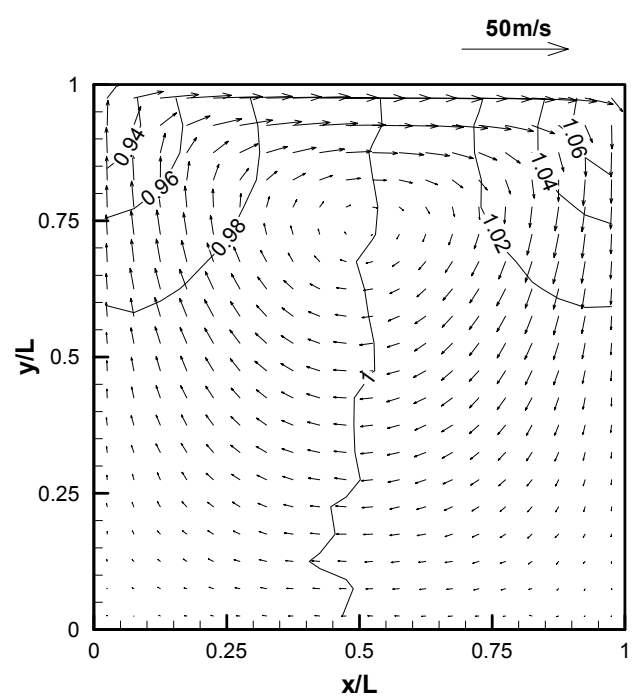

(b) DSMC

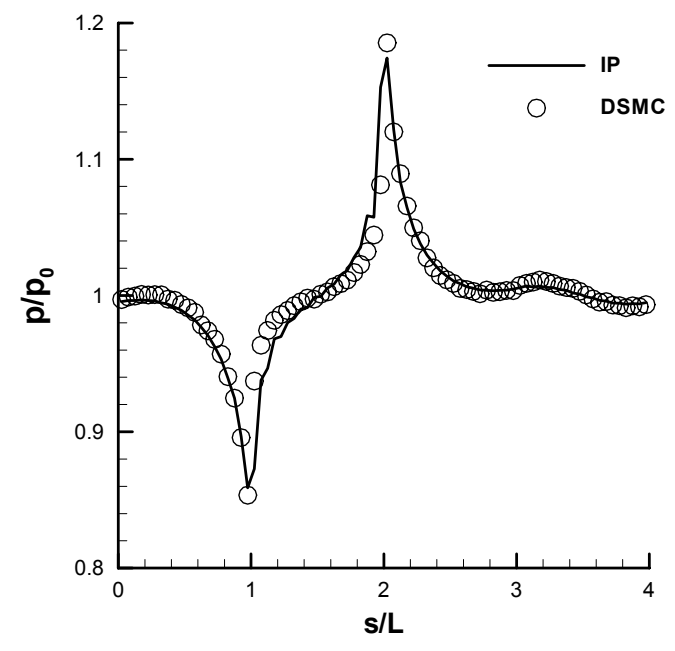

(c) Pressure distributions along the cavity surface

FIGURE 4. The IP and DSMC normalized density and velocity fields, and the normalized surface pressure distributions at $\mathrm{Ma}=0.14$, and $\mathrm{Kn}=0.1$. The IP and DSMC sample sizes are $2 \times 10^{5}$ and $5 \times 10^{6}$, respectively. 


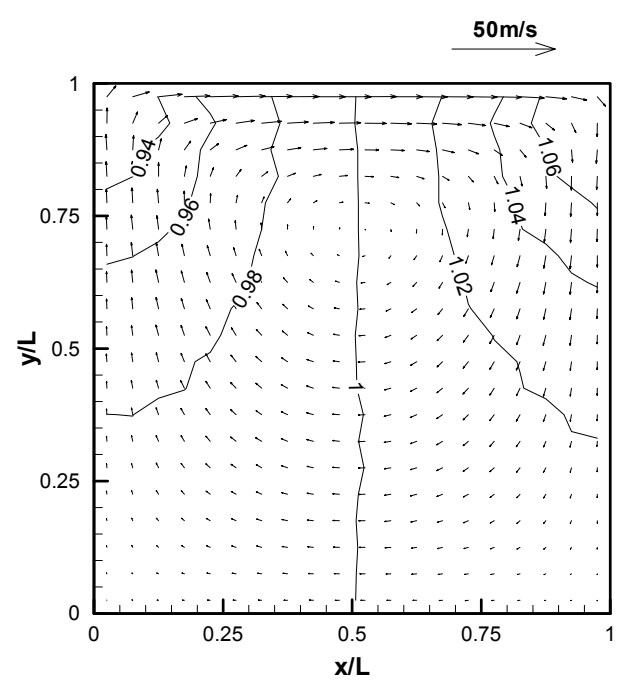

(a) IP

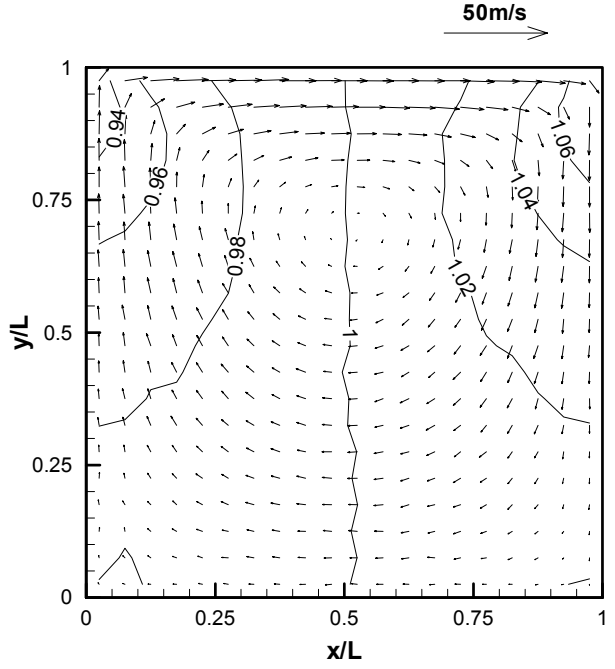

(b) DSMC

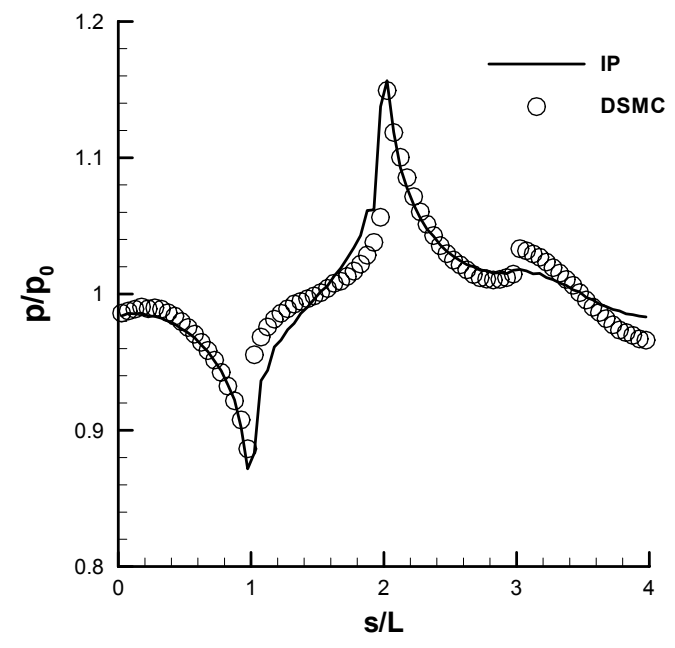

(c) Pressure distributions along the cavity surface

FIGURE 5. The IP and DSMC normalized density and velocity fields and normalized surface pressure distributions at $\mathrm{Ma}=0.14$, and $\mathrm{Kn}=1.0$. The IP and DSMC sample sizes are $2 \times 10^{5}$ and $5 \times 10^{6}$, respectively.

For the case of $K n=1.0$ and $M a=0.14$, the IP and DSMC results on the whole are still in agreement (Fig.5), whereas some local details are clearly different. For example, at the angle point $(s / L$ of 3$)$, there appears a discontinuity in the surface pressure distribution of DSMC, whereas the IP profile varies relatively gently. Comparison of the flow fields at the different Knudsen numbers shows that the influence region of an angle point significantly enlarges as $K n$ increases, e.g. the distance of the isopycnic line 1.02 from the angle point $\mathrm{C}$ is obviously larger at $\mathrm{Kn}=1.0$ (Figs. 5a and 5b) than at $\mathrm{Kn}=0.1$ (Figs. $4 \mathrm{a}$ and $4 \mathrm{~b}$ ).

Figure 6 shows the normalized density fields given by the IP and DSMC method for a case of $\mathrm{Kn}=0.1$ and $\mathrm{Ma}=2.8 \times 10^{-3}\left(u_{w}=1.0 \mathrm{~m} / \mathrm{s}\right)$. In contrast to the serious statistical scatter of DSMC at a huge sample size of 
$2 \times 10^{7}$, the IP method yield the statistically convergent result at a sample size of $2 \times 10^{5}$. The Mach number dependence of rarefied gas flow patterns may be seen through comparison of the density fields at Ma of 0.15 and $2.8 \times 10^{-3}$ (Figs. 4a and 6a).

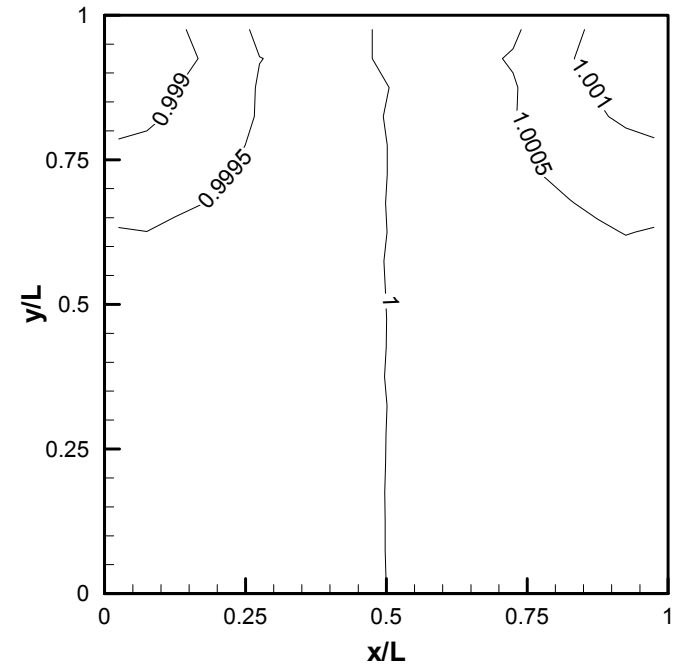

(a) IP

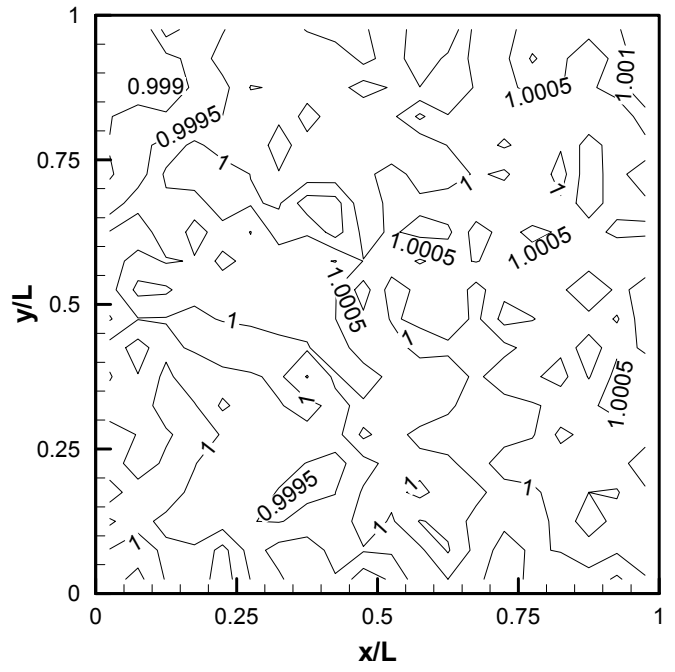

(b) DSMC

FIGURE 6. Normalized density fields given by the IP and DSMC method at $\mathrm{Ma}=2.8 \times 10^{-3}$, and $\mathrm{Kn}=0.1$. The IP and DSMC sample sizes are $2 \times 10^{5}$ and $2 \times 10^{7}$, respectively.

\section{ACKNOWLEDGEMENT}

The authors appreciate support from NNSFC under Grants 59876043 and 19889209.

\section{REFERENCES}

1. Bird, G. A., Molecular Gas Dynamics and the Direct Simulation of Gas Flows, Clarendon Press, Oxford, 1994.

2. Piekos, E.S., and Breuer, K.S., J. Fluids Eng. 118, 464 (1996).

3. Oh, C.K., Oran, E.S., and Sinkovits, R.S., J. Thermophysics. and Heat Transfer 11, 497 (1997)

4. Nance, R.P., Hash, D., and Hassan, H.A., J. Thermophysics and Heat Transfer 12, 447 (1997).

5. $\quad$ Ho, C.M., and Tai, Y.C., Annu. Rev. Fluid Mech. 30, 579 (1998).

6. Hadjiconstantinou, N. G., and Garcia, A. L. "Statistical Error in Particle Simulations of Low Mach Number Flows" in Proceedings of the First MIT Conference on Computational Fluid and Solid Mechanics, Elsevier, 2001.

7. Oran, E.S., Oh, C.K., and Cybyk, Z.C., Annu. Rev. Fluid Mech. 30, 403 (1998).

8. Millikan, R. A. Phys. Rev. 22, 1 (1923).

9. Tsien, H. S. ,J. Aero. Sci. 13, 653 (1946).

10. Fan, J., and Shen, C., "Statistical Simulation of Low-Speed Unidirectional Flows in Transition Regime" in Rarefied Gas Dynamics, edited by R. Brun et al, Cepadues Editions, 1999, Vol. 2, pp. 245.

11. Fan, J., and Shen, C., J. Comp. Phys. 167, 393 (2001). 
12. Cai, C., Boyd, I. D., Fan, J., and Candler G. V., J. Thermophysics and Heat Transfe 14, 368 (2000).

13. Fan, J., Boyd, I. D., and Cai, C. P., AIAA J. 39, 618 (2001).

14. Sun, Q., Boyd, I. D., and Candler, G. V., Numerical simulation of gas flow over micro-scale airfoils, AIAA 2001-3071 (2001).

15. Sun, Q.H., and Boyd, I.D., J. Comp. Phys. 179, 400 (2002).

16. Xie, C., Fan, J., and Shen, C., “ Rarefied Gas Flows in Micro-Channels” presented at this symposium.

17. Shen, C., Rarefied Gas Dynamics (in Chinese), Defense Industry Press, Beijing (to appear).

18. Sone, Y., Takata, S., and Ohwada, T., European J. Mech., B/Fluids 9, 273 (1990).

19. Ohwada, T., Sone, Y., and Aoki, K., Phys. Fluids 1, 2042 (1989).

20. Dong, W., University of California Report UCRL-3353 (1956).

21. Schaff, S. A., and Sherman, F. S., J. Aero Sci. 21, 85 (1954).

22. Janour, Z., Resistances of a plate in parallel flow at low Reynolds numbers, NACA TM 1316 (1954).

23. Pong, K. C., Ho, C. M., Liu, J. Q., and Tai, Y. C., ASME-FED 197, 51 (1994).

24. Shih, J. C., Ho, C. M., Liu, J. Q., and Tai, Y. C., ASME-DSC 59, 197 (1996).

25. Arkilic, E. B., Schmidt, M. A., and Breuer, K. S., "Measurement of the TMAC in silicon micro-channels" in Rarefied Gas Dynamics, edited by C. Shen, Peking University Press, Beijing, 1997, pp.983.

26. Ghia, U., Ghia, K. N., and Shin, C. T., J. Comp. Phys. 48, 387 (1982)

27. Su, M. D., Xu, K., and Ghidaoui, M. S., J. Comp. Phys. 150, 17 (1999)' 